\title{
Fangjifuling Ameliorates \\ Lipopolysaccharide-Induced Renal Injury via Inhibition of Inflammatory and Apoptotic Response in Mice
}

\author{
Zhonghao Su ${ }^{\mathrm{a}}$ Ping Yu $\mathrm{u}^{\mathrm{b}} \quad$ Lanyue Sheng $^{\mathrm{a}} \quad$ Jin Ye $\mathrm{C}^{\mathrm{a}} \quad$ Zhenxia Qin $^{\mathrm{a}}$ \\ aSchool of Basic Medicine, Shanghai University of Traditional Chinese Medicine, Shanghai, 'bnstitute of \\ Interdisciplinary Integrative Medicine Research, Shanghai University of Traditional Chinese Medicine, \\ Shanghai, China
}

\author{
Key Words \\ Fangjifuling (FF) • AKI • LPS • Apoptosis • Cell junction
}

\begin{abstract}
Background/Aims: Acute kidney injury (AKI) is a frequent and serious complication of sepsis; however, there is no effective treatment for it. FangJifuling (FF) decoction is widely used to treat acute glomerulonephritis and nephritic syndrome in the clinical setting. Methods: On the basis of its anti-inflammatory properties, the renoprotective effect of FF on a mouse model of lipopolysaccharide (LPS)-induced AKI was investigated. Major compounds were identified in FF with high-performance liquid chromatography. A bioinformatics analysis tool was used to predict target genes. Quantitative real-time PCR and western blot analyses were performed to validate the targets. Furthermore, the expression of a target gene was silenced by small interfering RNA-mediated knockdown in vitro. Results: Bioinformatics analysis indicated that inflammation, apoptosis, and cell junction were closely related to the renoprotective effects of FF. Validation was confirmed by an in vivo test. A reduction of inflammatory cell infiltration and inflammatory cytokine mRNA expression (iNOS, NF-KB, MCP-1, and TNF- $\alpha$ ) following the administration of $F F(50 \mathrm{mg} / \mathrm{kg})$ was observed in LPS-treated renal tissue. In addition, $\mathrm{FF}$ treatment suppressed mitochondrial-mediated apoptosis by regulating the $\mathrm{Bax} / \mathrm{Bcl}-2$ ratio in LPS-induced renal injury. Silencing $\mathrm{Cx} 43$, a cell-to-cell junction protein, was found to enhance the protective effect of FF against LPS-induced renal injury. Conclusion: Our study suggests that FF exhibits a renoprotective effect against LPS-induced inflammatory and apoptotic responses. In addition, Cx43 might be involved in these processes. These findings indicate the potential role of $\mathrm{FF}$ as a natural renoprotective product.
\end{abstract}




\section{Cellular Physiology Cell Physiol Biochem 2018;49:2124-2137 and Biochemistry Published onlme: 215eptember $2018 \begin{aligned} & \text { DOI: } 2018 \text { The Author(s). Published by S. Karger AG, Basel } \\ & \text { www.karger.com/cpb }\end{aligned}$}

Su et al.: Amelioration of LPS-Induced Renal Injury by Fangjifuling

\section{Introduction}

Acute kidney injury (AKI) is described as a significant worsening of kidney function [1]. Lipopolysaccharide (LPS) challenge has been used widely to produce animal models for studying the mechanisms underlying sepsis-induced AKI and its potential treatment.

The general inflammation mechanism for AKI is a reduction in renal perfusion, direct insults to the renal tubules, tubule-interstitial inflammation, and edema [2]. Kidney injury molecule-1 (KIM-1) and neutrophil gelatinase-associated lipocalin (NGAL) have been indicated as markers of AKI $[3,4]$. Tubular proximal cell apoptosis is a prominent and characteristic feature of AKI-induced renal inflammation and has been considered to be one of the mechanisms leading to renal injury [5].

FangJiFuling (FF) decoction is a traditional anti-inflammatory herb medicine used in China, firstly document as "Jing Gui Yao Lue" by Zhongjing Zhang during the Han Dynasty. The formula consists of five herbs: Stephania tetrandra S.Moore (Fangji), Astragalus propinquus Schischkin (Huangqi), Cinnamomum cassia (Nees \& T.Nees) J.Presl (Guizhi), Glycyrrhiza uralensis Fisch. (Gancao), and Poria cocos (Schwein.) F.A.Wolf (Fuling). Traditional Chinese Medicine (TCM) has been used for thousands of years to treat acute nephritis, rheumatism, cardiac failure, post-thrombotic syndrome, and other inflammation-related diseases [6-8]. The significant increase of urinary excretion and inhibition of the production inflammatory cytokines might be involved in the protective effect of FF on renal function. However, no report has described the mechanism by which FF alleviates renal injury induced by LPS.

The aim of this study was to investigate the effect of FF on LPS-induced AKI in mice. We hypothesized that FF would promote the reduction of kidney inflammation. However, little is known about its mechanism of action as an anti-inflammatory agent, which prompted us to investigate the effects of FF on the release of inflammatory mediators and the production of cytokines in a mouse model of LPS-induced AKI to understand its underlying mechanisms in the treatment of AKI.

\section{Materials and Methods}

\section{Preparation of FF}

Concentrated powders for FF were produced by Hongqiao Pharmaceutical Co., Ltd. (Shanghai, China) under internationally certified Good Manufacturing Practices guidelines. All plants and raw herbs were identified and authenticated by experienced botanists and chemists using thin-layer chromatography and high-performance liquid chromatography (HPLC).

The powders were decocted in $4 \mathrm{~L}$ distilled water twice for $30 \mathrm{~min}$ each. The two batches of filtrates were mixed together and centrifuged to remove herbal fragments. Extracts were concentrated to $1 \mathrm{~g} / \mathrm{mL}$ for use. Then, the extracts were concentrated to $1 / 10$ in a $37^{\circ} \mathrm{C}$ rotary evaporator after filtration under vacuum. The samples were freeze-dried, ground to a fine powder, and stored at $4^{\circ} \mathrm{C}$ until use. The preparation of components in the decoction was the same for each herb.

\section{Quantitative analysis and identification of the components of FF}

FF was analyzed using HPLC. A moderate amount of the samples was dissolved in $70 \%$ ethanol and then filtrated through a $0.45-\mu \mathrm{m}$ filter unit. Quantitative analysis of the composition of FF was performed on a 1200 series HPLC device (Agilent Technologies, Santa Clara, CA). Separation was performed using a GPC18 column ( $4.6 \times 250 \mathrm{~mm}$; particle size, $5 \mu \mathrm{m}$; Sepax, Newark, DE $)$ at $30^{\circ} \mathrm{C}$ during the analysis. The mobile phase consisted of $0.2 \%$ formic acid solution (A) and acetonitrile (B) with gradient elution as follows: 0-15 $\min (0-15 \%$ B), $15-20 \min (15-18 \%$ B), 20-30 min (18-20\% B), and 30-45 min (20-40\% B). The flow rate was set at $1.0 \mathrm{~mL} / \mathrm{min}$ and the signal was detected at $260 \mathrm{~nm}$. The injection volume was $10 \mu \mathrm{L}$. Data were acquired and processed using Chemstation software. 


\section{Cellular Physiology Cell Physiol Biochem 2018;49:2124-2137

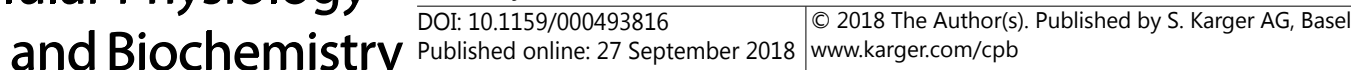

Su et al.: Amelioration of LPS-Induced Renal Injury by Fangjifuling

Acute kidney injury model

C57BL/6J mice, 8-12 weeks of age (18-22 g), were used in this study. They were housed in an airconditioned room with a 06:00 to 18:00 light cycle and fed standard rodent chow. All experiments and animal care were approved by the Animal Research Ethics Committee at Shanghai University of TCM.

To assess mortality rates, the mice were given either LPS (Escherichia coli 0111:B4; Sigma) or an equivalent volume of endotoxin-free $0.9 \%$ saline vehicle by intraperitoneal (IP) injection [9]. The mice were divided randomly into four groups ( $=20$ per group): (1) Control; (2) LPS (20 mg/kg); (3) LPS + FF (25 $\mathrm{mg} / \mathrm{kg}$ ); and (4) LPS + FF (50 mg/kg). FF was administered orally for 2 weeks before LPS stimulation. At 1 $\mathrm{h}$ after LPS challenge, the mice were given either FF or vehicle (equal volume saline) by oral administration. Mortality was recorded every $6 \mathrm{~h}$ for $48 \mathrm{~h}$ after LPS injection in each group.

For all other experiments, the mice were divided randomly into four groups ( $\mathrm{n}=10$ per group): (1) Control; (2) LPS (10 mg/kg); (3) LPS + FF (50 mg/kg); and (4) FF (50 mg/kg). After sacrifice at 48 h, kidney tissue and blood were collected.

\section{Biochemical and histology studies}

Serum creatinine (SCR), blood urea nitrogen (BUN), aspartate aminotransferase (AST), and alanine aminotransferase (ALT) levels were measured with kits from Nanjing Jianchen. Tissues were cut into 5- $\mu \mathrm{m}$ sections and stained with hematoxylin and eosin (H\&E). According to the proportions of necrotic tubules and cast formation relative to the total number of tubules, tubular injury in the cortex and outer medulla was graded into five levels (1: 10\%; 2: 10-25\%; 3: 25-50\%; 4: 50-75\%; 5: 75\%).

\section{Immunofluorescence assays}

For the immunofluorescent analysis of connexin 43 ( $\mathrm{Cx} 43)$, kidney tissues were embedded in OCT, snap frozen in liquid nitrogen, and stored at $-80^{\circ} \mathrm{C}$ until sectioning. Cryostat sections $(5-\mu \mathrm{m}$ thick) were fixed in $4 \%$ paraformaldehyde and prepared for immunofluorescence studies. The primary antibody used was an anti-mouse Cx43 monoclonal antibody (D120496; Sangon, China). DAPI was used to label nuclei.

\section{Quantitative real-time PCR}

Total RNA was extracted from kidney tissue with the traditional TRIzol method. The concentration and purity of RNA were measured on a Take3 Synergy ${ }^{\mathrm{TM}} 2$ (Biotek, Broadview, IL). Reverse transcription and quantitative real-time (qRT-PCR) were performed on an ABI (Applied Biosystems, Foster City, CA) with a Takara kit (Takara Bio Inc., Kusatsu, Japan; 036a, 420a). The primers were as follows: iNOS 5'-AGGGAATCTTGGAGCGAGTT-3' and 5'-TGGACGACTCTTGGGAGAAG-3'; NF- $\kappa B$ 5'-TGGACGACTCTTGGGAGAAG-3' and 5'-GAACACAGGCTCATACGGTTTC-3'; MCP$1 \quad 5^{\prime}$-CAGATCTCTCTTCCTCCACCACTAT-3 ${ }^{\prime}$ and $5^{\prime}$-CAGGCAGCAACTGTGAACAAC- $3^{\prime}$; $\quad$ TNF- $\alpha$ 5'-TGATCGGTCCCAACAAGGA-3' and ${ }^{\prime}$ '-TGCTTGGTGGTTTGCTACGA-3'; and GAPDH 5'-CCCCCAATGTATCCGTTGTG- ${ }^{\prime}$ ' and 5'-TAGCCCAGGATGCCCTTTAGT- ${ }^{\prime}$. All results were obtained from at least three independent experiments. The data were calculated using the $2^{-\Delta \Delta \mathrm{CT}}$ method and normalized to GAPDH as an internal control.

\section{Western blot analysis}

Western blot analysis was carried out as described previously [9]. An equal amount of protein was separated by sodium dodecyl sulfate-polyacrylamide gel electrophoresis and transferred to and immobilized on a polyvinylidene fluoride membrane. After blocking with $5 \%$ fat-free milk for $1 \mathrm{~h}$, the membrane was blotted overnight at $4^{\circ} \mathrm{C}$ with the following primary antibodies: anti-NGAL (Santa Cruz Biotechnology, Santa Cruz, CA; sc-50351) anti-KIM-1 (Abcam, Cambridge, UK; ab47635), anti-Bax (Cell Signaling Technology, Danvers, MA; \#2772), anti-Bcl-2 (Cell Signaling Technology; \#2870), anti-Cox-2 (Cell Signaling Technology; \#4842), anti-IL-1 $\beta$ (Cell Signaling Technology; \#8689), anti-NF-кB p65 (Cell Signaling Technology; \#8242), anti-cleaved caspase-3 (Cell Signaling Technology; \#9661), and anti-GAPDH (GeneTex, Inc., Irvine, CA; GTX100118). The membrane was incubated with horseradish peroxidase-conjugated secondary antibodies (Santa Cruz Biotechnology) at room temperature for $1 \mathrm{~h}$. Pierce ECL Western Blotting Substrate (Thermo Fisher Scientific, Waltham, MA) was used to detect antigen-antibody complexes. Densitometric quantification was performed with an image analysis program (Tanon-2500, Tanon, Shanghai, China). 


\section{Cellular Physiology Cell Physiol Biochem 2018;49:2124-2137 and Biochemistry \begin{tabular}{l|l} 
DOI: 10.1159/000493816 & $\begin{array}{l}\text { C } 2018 \text { The Author(s). Published by S. Karger AG, Basel } \\
\text { www.karger.com/cpb }\end{array}$
\end{tabular}

\section{Prediction of target genes for $F F$}

We selected the BATMAN-TCM database as a bioinformatics analysis tool (http:// http://bionet.ncpsb. org/batman-tcm/)[1]. FF is composed of five herbs: Astragalus propinquus, Stephania tetrandra, Poria cocos. Glycyrrhiza uralensis, and Cinnamomum cassia; the Pinyin names were input for all of the herbs, denoted by "HUANG QI; FANG JI; FU LING; GAN CAO; GUI ZHI," respectively. Predicted target genes (including known targets) with scores higher than 20 for each ingredient are presented and used for further bioinformatics analyses.

Fifty-seven target genes of FF were analyzed with Gene Ontology (GO) and pathway enrichment analysis with DAVID Bioinformatics Resources 6.8 (http://david.abcc.ncifcrf.gov/). Enriched GO terms (pathways) with a P-value less than 0.05 (corrected with Bonferroni's "step-down") were collected and analyzed to interpret the biological function of these target genes using the functional annotation tools of DAVID and the Kyoto Encyclopedia of Genes and Genomes (KEGG).

\section{Cell Culture}

HK-2 cell culture was performed as reported previously [9]. In short, the cells were maintained in Dulbecco's modified Eagle's medium/F12 medium containing 10\% heat-inactivated fetal bovine serum, $1 \%$ penicillin-streptomycin, pH 7.4 , at $37{ }^{\circ} \mathrm{C}$ in a humidified $5 \% \mathrm{CO}_{2}$ incubator. The cells were passaged by trypsinization every 3-4 days.

\section{Cell Counting Kit-8 assay}

Cell viability was measured as described previously [10]. Briefly, $10 \mu \mathrm{L}$ Cell Counting Kit-8(CCK-8) (Beyotime Biotechnology,China) solution was added to each well and incubated for $30 \mathrm{~min}$ at $37{ }^{\circ} \mathrm{C}$. Absorbance was measured at $450 \mathrm{~nm}$ with a microplate reader (BioTek Instruments ELX800, USA).

\section{Transfection of small interfering RNA}

Human Cx43 small interfering RNA (siRNA) and negative control were synthesized by GenePharma (Shanghai, China). siRNA sequences for Cx43 were (forward) 5'-AGUACGGUAUUGAAGAGCAUGGUAA3' and (reverse) 3'-UUACCAUGCUCUUCAAUACCGUACU-5'. siRNA transfection was performed using Lipofectamine ${ }^{\mathrm{TM}}$ according to the manufacturer's instructions.

\section{Statistical analysis}

All data are expressed as the mean \pm standard deviation (SD) and compared using the Mann-Whitney U-test and one-way analysis of variance with SPSS 19.0 (IBM, Armonk, NY). Statistical significance was considered when a P-value was less than 0.05 .

\section{Results}

\section{Identification of the major compounds in FF with HPLC}

To ensure the consistency and quality control of FF manufacture, the marker compounds from FF (Table 1) were measured with chromatographic technologies. Major compounds were identified in FF with HPLC (Fig. 1A, Table 2). By referring to the standards, the chromatographic analysis showed that the main components of FF were tetrandrine, calycosin-7-glucoside, ammonium glycyrrhetate, and cinnamaldehyde (Fig. 1B).

Table 1. The ratio of the components in FF

\begin{tabular}{lcc}
\hline Latin name & Pinyin name & Ratio \\
\hline Stephania tetrandra S. Moore (family: Menispermaceae) & Fangji & 1 \\
Astragalus propinquus Schischkin (family: Leguminosae) & Huangqi & 1 \\
Cinnamomum cassia (Nees \& T.Nees) J.Presl (family: Lauraceae) & Guizhi & 1 \\
Glycyrrhiza uralensis Fisch. (family: Leguminosae) & Gancao & 1 \\
Poria cocos (Schw.) Wolf (family: Polyporaceae) & Fuling & 2 \\
\hline
\end{tabular}


FF improves the survival, renal function, and biomarkers of mice with LPS-induced AKI

The death rate of LPS-induced mice was reduced significantly with FF treatment. Cumulative mortalities were observed over $48 \mathrm{~h}$ in the high-dose FF group (50 mg/kg; 4/20; $20 \%$ ) (compared with the LPS group, $\mathrm{P}<0.05$ ), lowdose FF group $(25 \mathrm{mg} /$ $\mathrm{kg} ; 6 / 20 ; 30 \%)$, and LPS treatment group $(20 / 20$; $100 \%$ ) (compared with the control group, $\mathrm{P}<0.05$ ) (Fig. 2A).

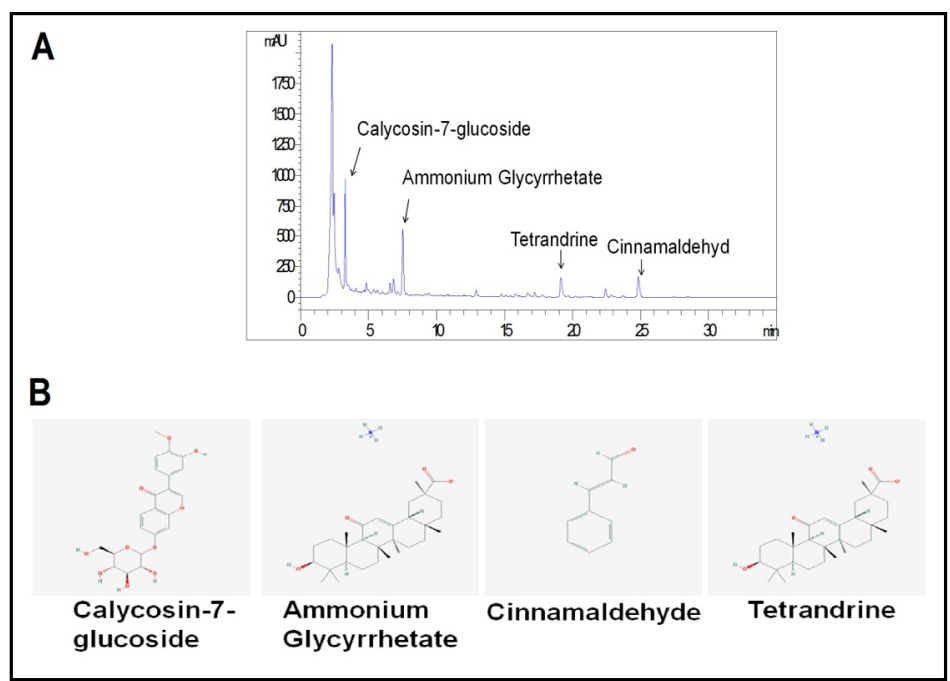

Fig. 1. Identification of compounds in FF with HPLC. A. Fingerprint analysis of FF using HPLC. B. The reference compounds of FF were identified by HPLC analysis.

Table 2. Components of FF analysized with HPLC

\begin{tabular}{lcccc}
\hline & Calycosin-7-glucoside & Ammonium Glycyrrhetate & Tetrandrine & Cinnamaldehyde \\
\hline Retention time(min) & 3.25 & 7.53 & 19.83 & 24.56 \\
Mean(mg/g) & 65.2 & 45.3 & 18.0 & 17.2 \\
\hline
\end{tabular}

Fig. 2. Protective effects of $\mathrm{FF}$ on the development of LPS-induced AKI. A. The survival rate of 20 mice per group was observed every $6 \mathrm{~h}$. FF was administered orally for 2 weeks before LPS stimulation. The mice were challenged by LPS (20 $\mathrm{mg} / \mathrm{kg}$, IP) with or without FF $(25,50$ $\mathrm{mg} / \mathrm{kg}$, oral administration) treatment. B. The kidney-to-body weight ratio was measured. At $1 \mathrm{~h}$ after LPS challenge (10 $\mathrm{mg} / \mathrm{kg}$, IP), the mice were given either FF $(50 \mathrm{mg} / \mathrm{kg}$ ) or vehicle (same volume saline) by oral administration. Then, all mice were sacrificed at $48 \mathrm{~h}$. C-D. Changes in SCR and BUN for each group. E-H. Western blot analysis of the renal injury marker proteins $\mathrm{KIM}-1$ (E, F) and NGAL $(G, H)$ in kidney tissue at $48 \mathrm{~h}$ after LPS treatment. Data are expressed as the mean \pm SD with 8 mice per group. * $\mathrm{P}<0.05$, ${ }^{*} \mathrm{P}<0.05$.

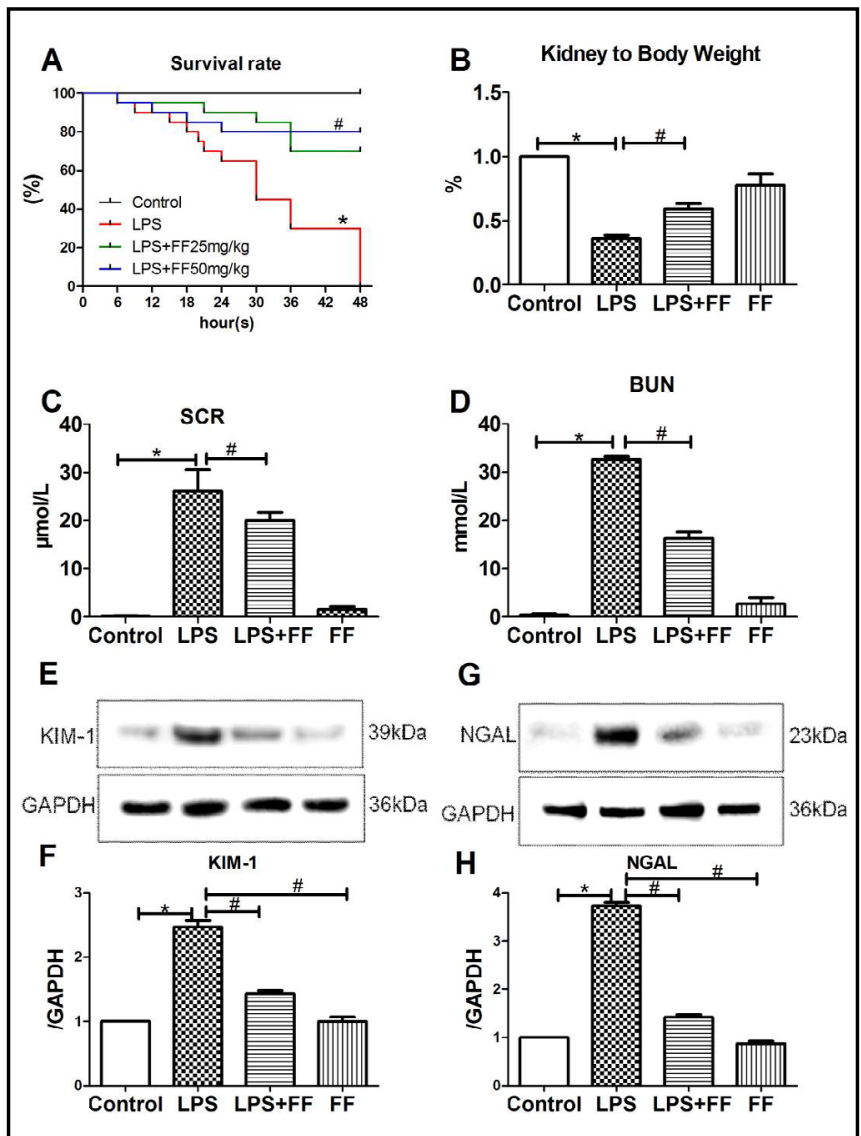




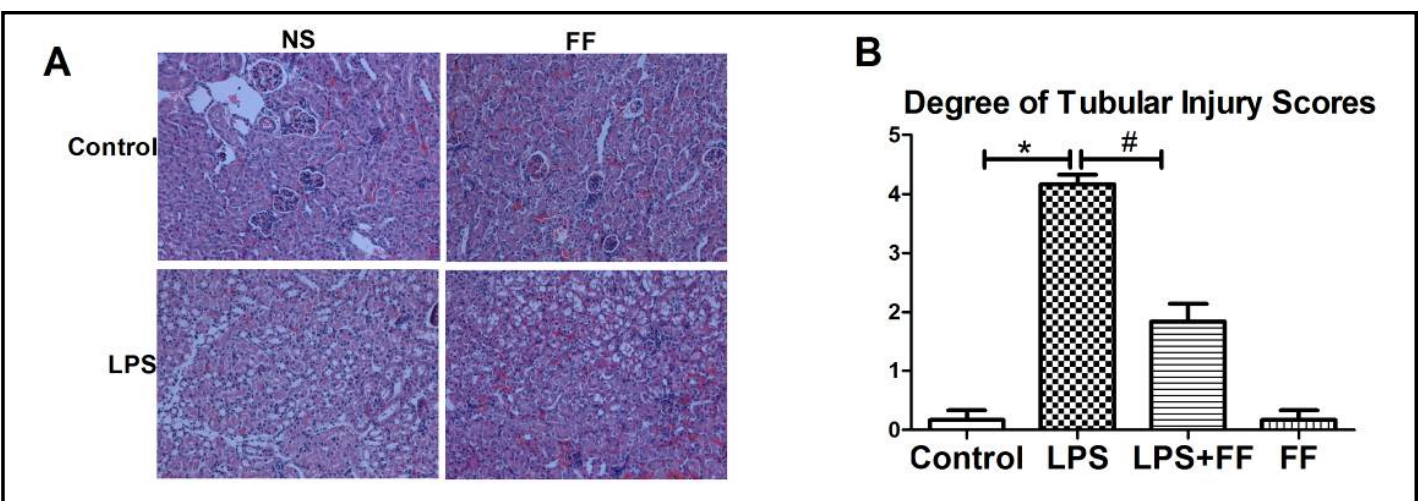

Fig. 3. Amelioration of LPS-induced histological changes by FF in renal tissue. A. Representative images of mouse renal tissue sections stained with H\&E at $48 \mathrm{~h}$ after LPS injection. B. Tubular injury was determined with semi-quantitative analysis. Magnification, $\times 200$. Data are expressed as the mean \pm SD with 8 mice per group. ${ }^{*} \mathrm{P}<0.05$, $\# \mathrm{P}<0.05$.

On the basis of the study of LPS toxicity, high-dose FF (50 mg/kg) was selected to study its protective effects on LPS (10 mg/kg)-induced AKI. Since urinary output was increased by LPS, the kidney-to-body weight ratio was measured. FF significantly restored renal weight compared with mice treated with LPS alone after $48 \mathrm{~h}$ (Fig. 2B). SCR (Fig. 2C) and BUN (Fig. 2D) levels were highly increased in the LPS group at $48 \mathrm{~h}$ compared with the control group $(P<0.05)$. The levels of SCR and BUN induced by LPS were significantly $(P<0.05)$ ameliorated by FF treatment, while they were not affected by FF alone. KIM-1 and NGAL were selected as early markers for the

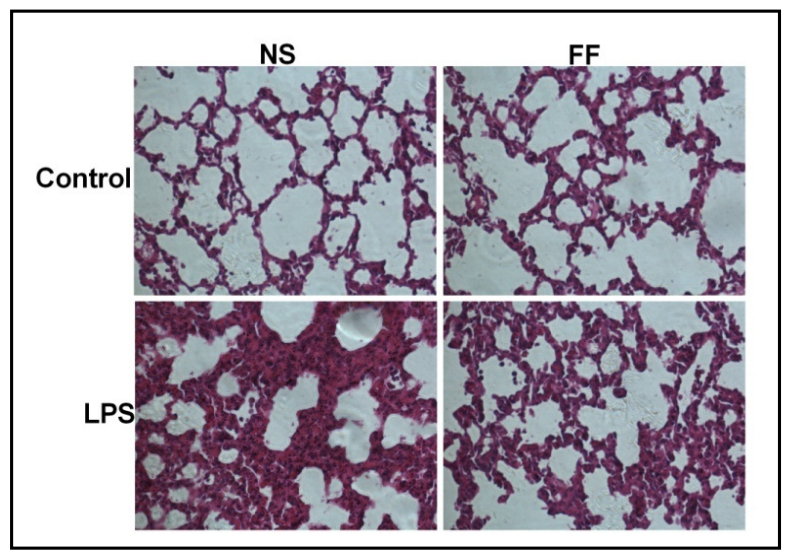

Fig. 4. FF ameliorates LPS-induced lung histopathological changes. Representative images of mouse lung tissue sections stained with H\&E at $48 \mathrm{~h}$ after LPS injection. Magnification, $\times 100$. deterioration of the renal function index and used to predict AKI. LPS administration significantly increased KIM-1 and NGAL protein expression in renal tissues, while FF attenuated their expression (Fig. 2E-H).

\section{FF attenuates LPS-induced renal histopathology injury}

To determine the protective effects of FF on LPS-induced renal injury, structural changes in kidney tissue were evaluated. Renal tubules and corpuscles were normal with H\&E staining in sections from the control and FF groups. In contrast, vacuolar degeneration and inflammatory cell infiltration were observed in the LPS-treated group. Interestingly, co-administration of FF significantly improved the degenerative changes in the LPS-treated group (Fig. 3).

In addition, acute lung and liver injury induced by LPS was also attenuated by FF. Significant histopathological changes were observed including a large amount of neutrophil infiltration, alveolar wall edema, and alveolar hemorrhage with H\&E staining in LPS-treated pulmonary tissue. It was shown that FF played a protective role against lung injury (Fig. 4). FF inhibited LPS-induced hepatotoxicity as indicated by pathological changes including extensive and severe hemorrhagic necrosis (Fig. 5A) and diagnostic indicators of liver damage aspartate aminotransferase(AST) and alanine aminotransferase(ALT) levels (Fig. 5B).

\section{KARGER}




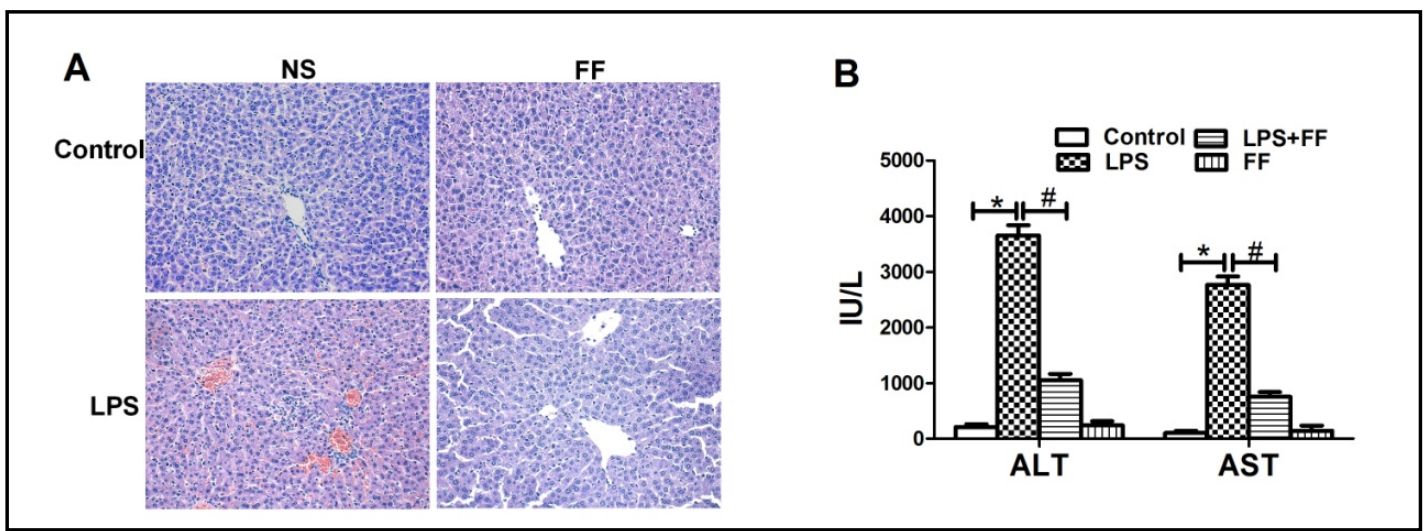

Fig. 5. Histopathological micrographs and liver function following LPS administration in the presence or absence of FF. A. Representative images of mouse liver tissue sections stained with H\&E at $48 \mathrm{~h}$ after LPS injection. Magnification, $\times 100$. B. Serum ALT and AST levels were measured by using corresponding assay kits according to the manufacturer's instructions. Blood was collected at $48 \mathrm{~h}$ after LPS injection.

\section{Bioinformatics analysis with BATMAN-TCM and the DAVID database}

To unveil the molecular mechanisms of FF renoprotection, using the BATMAN-TCM database (http://bionet.ncpsb.org/batmantcm/), the potential gene targets of the five herbs in FF (HUANG QI, FANG JI, GUI ZHI, FU LING, and GAN CAO) were predicted. In total, 2487 gene targets of FF were identified. There were 57 overlapping gene targets among the five herbs including ACHE, ADORA1, ADRA2B, ADRA2C, AKR1C3, ANXA1, AR, B4GALT1, BAX, BCHE, CALB1, CHRNA3, CHRNB2, CHRNB4, CYP24A1, CYP27A1, CYP27B1, CYP2R1, CYP3A4, DRD5, ESR1, F2RL1, FGF23, GC, GFI1, GPBAR1, HCN2, HCN4, HIF1A, HTR2A, HTR2B, IL1B, IRX5, KANK2, KCNMA1, KL, LANCL2, MED1, NFKB1, NOS1, NR1H4, NR3C1, OPRK1, PDE2A, PGR, PML, RAPGEF2, RXRA, S100G, SLC6A4, SNAI1, SNAI2, SNW1, TCF3, TRIM24, and VDR (Fig. 6A).

Using the Search Tool for the Retrieval of Interacting Genes online database (http:// string-db.org) [10] and Cytoscape software [11], a protein-protein interaction network was created with the target genes (Fig. 6B). The overrepresentation of GO categories was assessed using the Cytoscape plug-in BinGO. Hubs defined as nodes with more than 5 components were considered to play a critical role in the network [12], including NOS1, NF- $\kappa B$, and IL$1 \mathrm{~b}$, and are shown in Table 3. Using the DAVID database, GO analysis was performed with KEGG pathway analysis. Target genes were classified into three functional groups: molecular function, biological process, and cellular component. In KEGG pathway analysis, the genes were mainly involved in inflammatory mediator regulation of TRP channels, neuroactive ligand-receptor interaction, and endocrine and other factor-regulated calcium reabsorption (Fig. 6C). In the cellular component group, the genes were mainly enriched in cell junction, protein complex, and membrane (Fig. 6D). In the molecular function group, the genes were mainly enriched in protein binding, DNA binding, and transcriptional activator activity (Fig. 6E). In the biological process group, the genes were mainly enriched in the negative regulation of the apoptotic process, cellular response to LPS, and regulation of the Toll-like receptor signaling pathway (Fig. 6F). While pathway analysis indicated that the target genes of FF were mainly related to the regulation of inflammatory mediators. It is considered that the renoprotective effects of FF were achieved by inhibiting the production of inflammatory cytokines and tubular cell apoptosis, which will be investigated further in the following experiments. 


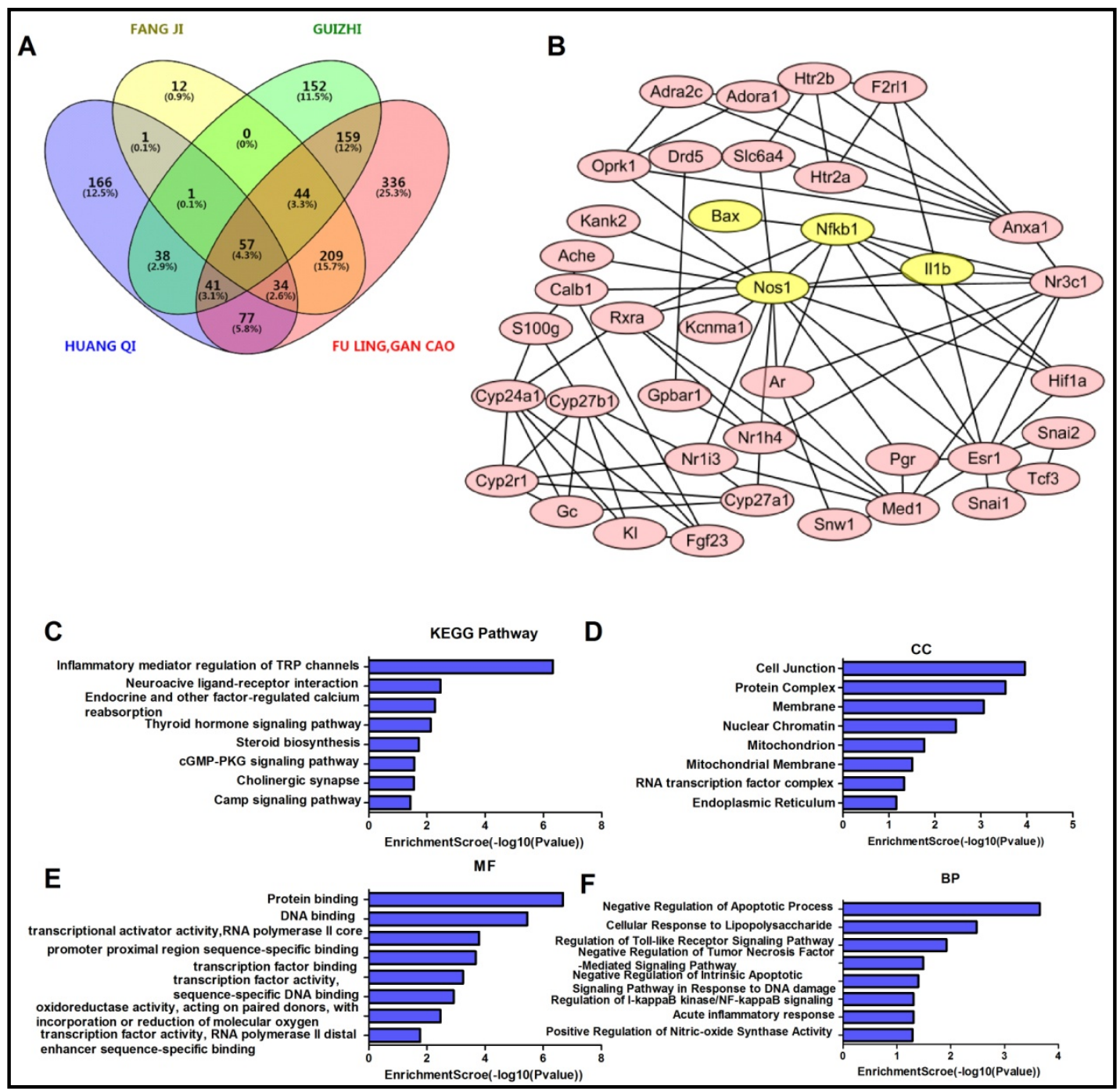

Fig. 6. Drug-target interaction network analysis in FF based on BATMAN TCM and DAVID database. A. Venn diagram showing overlapping target genes of the five herbs in FF predicted by BATMAN-TCM. HUANG QI: Astragalus Membranaceus; FANG JI: Stephania Tetrandra; FU LING: Poria cocos; GAN CAO: Glycyrrhiza uralensis. GUI ZHI, Cinnamomum Cassia. B. Protein-protein interaction (PPI) complex network originated from target genes. C-F. Gene ontology analysis (GO) were performed with DAVID bioinformatics resources. C. Top 8 significant changes KEGG pathway analysis. D. Top 8 significant changes cellular component (CC) analysis. E. Top 8 significant changes molecular function (MF) analysis. F. Top 8 significant changes biological process (BP) analysis.

\section{FF alleviates LPS-stimulated renal inflammatory response}

Considering that the predicted target genes analysis indicated that regulation of inflammatory might be one major relative pathways of FF, We focused on examining the expression of inflammatory cytokine. It was found that LPS significantly increased inflammatory cytokine expression including iNOS(one of the nitric oxide synthases), NF$\kappa B$, MCP- 1 , TNF- $\alpha$, IL-1 $\beta$ and COX-2 in the kidney tissues compared with the control group at $48 \mathrm{~h}$ after LPS injection $(\mathrm{P}<0.05)$. It is no wonder that FF could inhibit LPS-induced inflammatory cytokines production (Fig. 7). 
FF represses LPS-induced nephrocyte apoptosis

On the basis of protein-protein interaction network analysis, it was suggested that the anti-inflammatory effects of FF were probably related to the renal cell apoptotic process (Fig. 4B). We further measured the expression of the apoptotic-related proteins Bax and Bcl2. The Bax/Bcl-2 ratio increased by LPS was inhibited by FF. Clearly, the induction of renal cell apoptosis could be inhibited by FF (Fig. 8A, B).

FF alleviates tubular injury partly by regulating cell junction function

As mentioned in the GO analysis, the target genes involved in cell junction function were highly enriched in the cellular component group (Fig. 6E). One of the cell junction proteins expressed abundantly in renal tissue, namely, Cx43, was related to cellular apoptosis $[13,14]$. Immunohistochemistry showed that $\mathrm{Cx} 43$ was a major expressed protein around the proximal tubules. $\mathrm{Cx} 43$ was not detectable in the control and FF groups. However, Cx43 was positively expressed in the distal tubules in the LPS-treated group. Moreover, $\mathrm{Cx} 43$ was expressed at a lower level in the FF group than in the LPStreated group (Fig. 9A-C).

Table 3. the list of target genes node degree $\geq 6$

\begin{tabular}{lcc}
\hline Number & Gene name & Node degree \\
\hline 1 & Nos1 & 16 \\
2 & Esr1 & 9 \\
3 & Nfkb1 & 8 \\
4 & Med1 & 8 \\
5 & Nr3c1 & 8 \\
6 & Cyp24a1 & 7 \\
7 & Cyp27b1 & 7 \\
8 & Anxa1 & 6 \\
9 & Il1b & 6 \\
\hline
\end{tabular}

Fig. 7. Suppression of LPS-induced renal inflammatory cytokines by $\mathrm{FF}$ at the mRNA and protein expression level. A-D. Expression of iNOS, NF- $\kappa B$, MCP1 , and TNF- $\alpha$ genes in mouse kidney tissue was analyzed with qRT-PCR at the indicated times. E-H. The expression of the inflammatory proteins IL-1 $\beta$ (E, F) and $\operatorname{COX}-2(\mathrm{G}, \mathrm{H})$ with western blot analysis in kidney tissue at 48 $\mathrm{h}$ after LPS treatment. Data are expressed as the mean \pm SD with 8 mice per group. ${ }^{*} \mathrm{P}<0.05$, \#P<0.05.

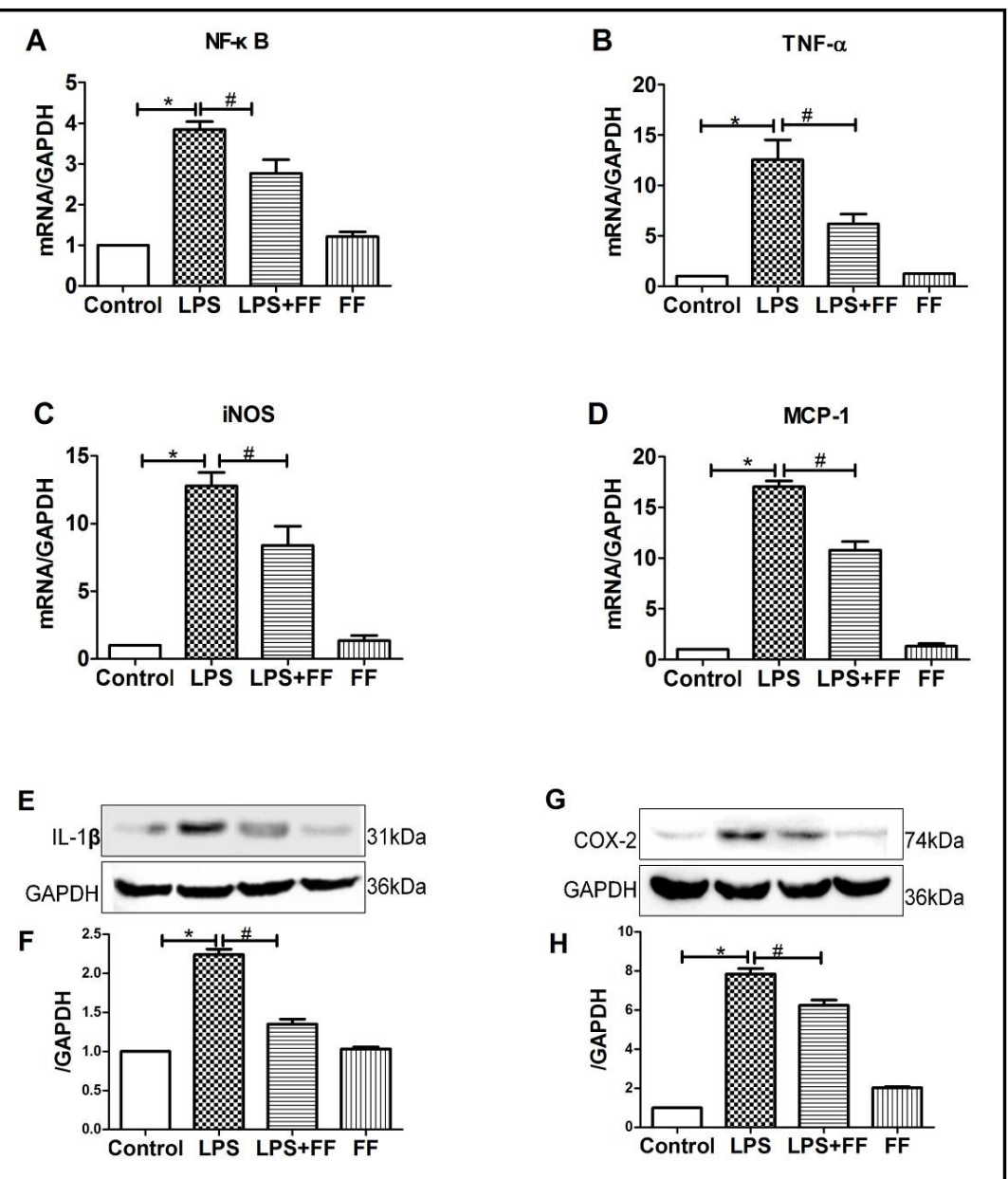




\section{\begin{tabular}{ll} 
Cellular Physiology & \multicolumn{1}{c}{ Cell Physiol Biochem 2018;49:2124-2137 } \\
DOI: 10.1159/000493816 & 2018 The Author(s). Published by S. Karger AG, Basel
\end{tabular}

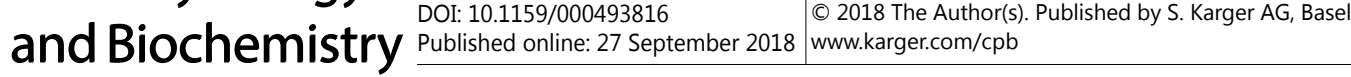

Silencing CX43 enhances the protective effects of FF against the inflammatory response and apoptosis stimulated by LPS in HK-2 cells

Considering that $\mathrm{Cx} 43$ was mainly present in tubular epithelial cells, human renal tubular epithelial HK-2 cells were stimulated by LPS as an in vitro model to investigate the role of Cx43 in the alleviation of LPS-induced renal injury by FF.

First, the cytotoxicity of FF was investigated in HK-2 cells with a CCK-8 assay. FF (10$200 \mu \mathrm{g} / \mathrm{mL}$ ) had no obvious impact on cell viability after treatment for $24 \mathrm{~h}$ (Fig. 10A). Furthermore, cell viability was also evaluated with FF treatment in the presence of LPS (1 $\mu \mathrm{g} / \mathrm{mL}$ ); however, no significant cytotoxicity was found with FF and LPS treatment (Fig. 10B).

To explore the role of $\mathrm{Cx} 43$ in the LPS-induced apoptotic and inflammatory response, either siRNA-Cx43 or negative control siRNA was transfected into HK-2 cells. The siRNA used could efficiently knock down Cx43 expression in HK-2 cells (Fig. 11A, B). To determine whether $\mathrm{Cx} 43$ siRNA could affect the protective effects of FF against the inflammation and apoptosis stimulated by LPS, HK-2 cells were stimulated with LPS in the presence or absence of $\mathrm{FF}$ as described above after transfection with siRNA against $\mathrm{Cx} 43$ or control siRNA. As shown in Fig. 11C, D, the inflammatory effects and apoptosis induced by LPS could be weakened by Cx43 knockdown, and the protective effects of FF against LPS-stimulated injury were enhanced by the siRNA-mediated knockdown of $\mathrm{Cx} 43$, while they were not affected by control siRNA.

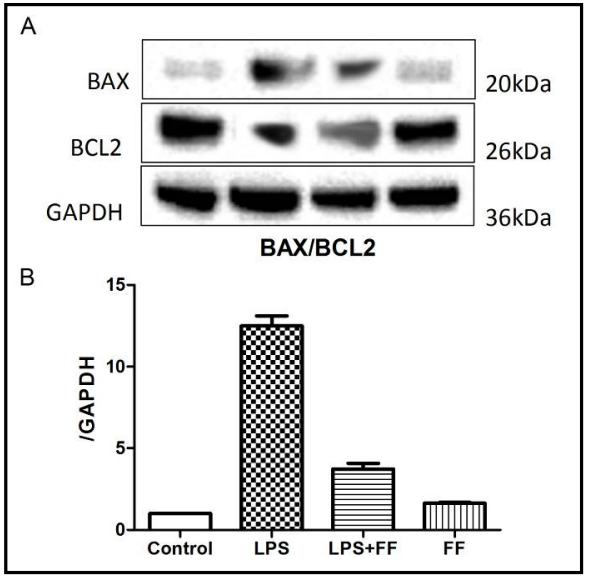

Fig. 8. Inhibition of LPS-induced renal cell apoptosis by FF. A. Representative images of the apoptotic-related proteins Bax and Bcl-2 in mouse kidney tissue. The same amounts of protein were loaded and GAPDH was used as a loading control. B. The Bax/Bcl2 ratio was calculated. Data are expressed as the mean $\pm \mathrm{SD}$ with 8 mice per group. ${ }^{*} \mathrm{P}<0.05$, ${ }^{\text {}} \mathrm{P}<0.05$.

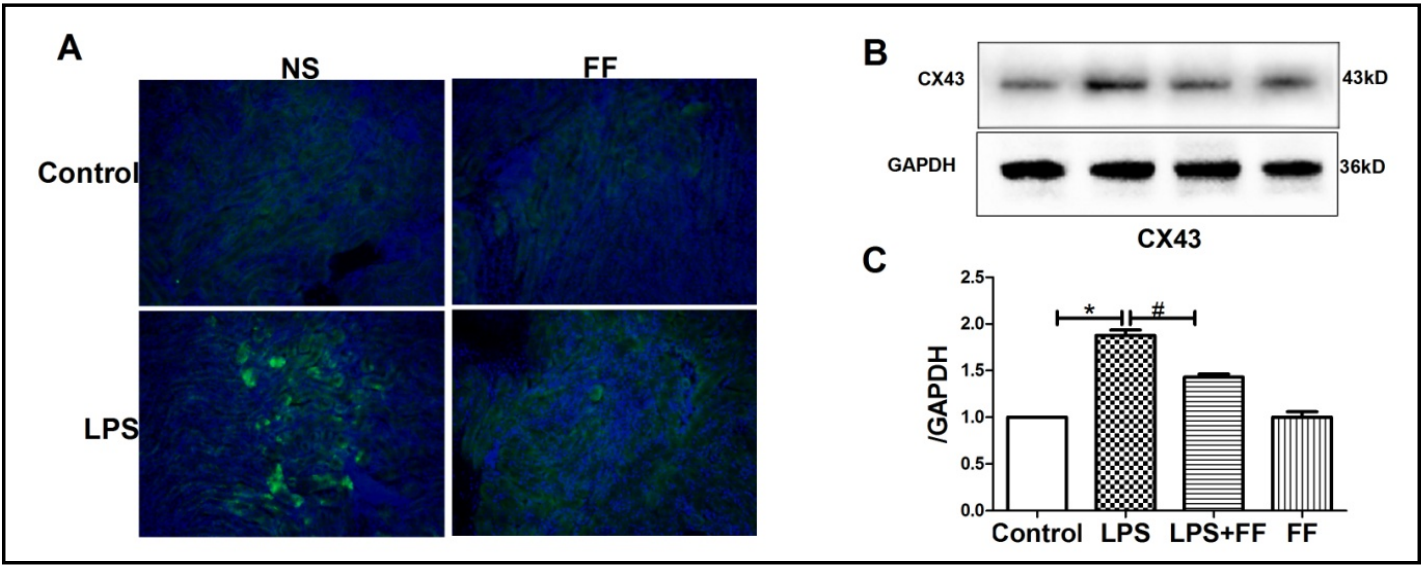

Fig. 9. Effect of FF treatment on $\mathrm{Cx} 43$ expression in mouse kidney. A. Representative images showing the expression and localization of $\mathrm{Cx} 43$ (green) and DAPI (blue) by immunofluorescent staining in renal tissue sections $(100 \times)$. B. Western blot analysis of $\mathrm{Cx} 43$ protein levels from mouse kidney. C. The data are presented after normalization to GAPDH expression and shown as the mean \pm SD with 8 mice per group. ${ }^{*} \mathrm{P}<0.05$, " $\mathrm{P}<0.05$. 
Fig. 10. Cytotoxicity of FF and LPS in HK-2 cells. A. Cell viability of HK-2 cells was measured after incubated with FF (10-200 $\mu \mathrm{g} / \mathrm{mL}$ ) for $24 \mathrm{~h}$. B. Cell viability of HK-2 cells was measured after treated with FF at different concentrations in the presence of LPS $(1 \mu \mathrm{g} / \mathrm{mL})$ for $24 \mathrm{~h}$.
B

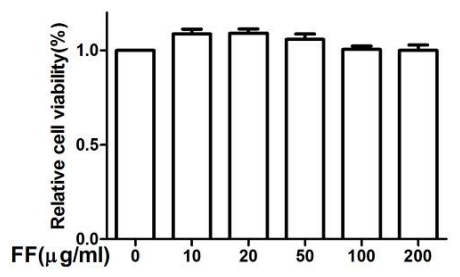

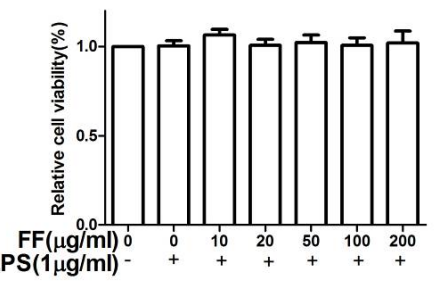

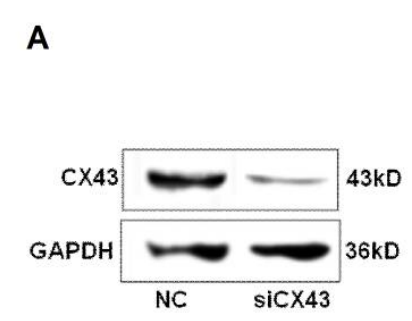

B

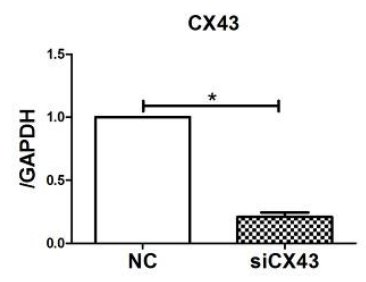

C

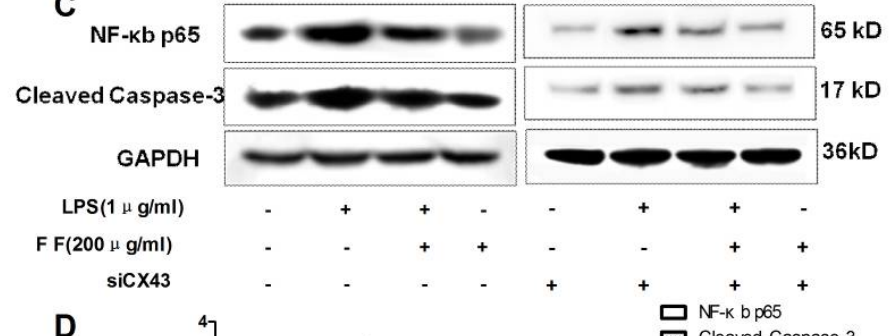

D

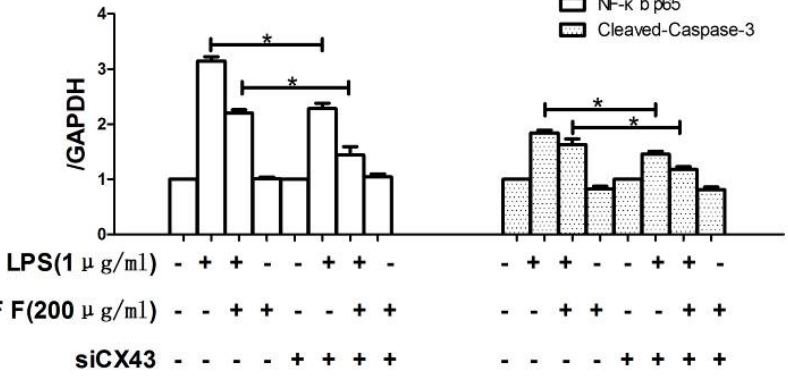

Fig. 11. Effects of $\mathrm{Cx} 43$ downregulation on the protective effects of $\mathrm{FF}$ against the inflammation and apoptosis induced by LPS in HK-2 cells. A, B. Knockdown of Cx43 by siRNA was determined by western blot analysis. HK-2 cells were transfected with Cx43-siRNA or negative control siRNA (NC siRNA). The expression levels of $\mathrm{Cx} 43$ in the transfected cells were assessed by western blot analysis and shown as the mean \pm standard error of the mean of three independent experiments. C, D. The expression levels of NF- $\kappa \mathrm{B}$ and cleaved caspase- 3 were measured with western blot analysis. After transfection with siRNA against $\mathrm{Cx} 43$ for $48 \mathrm{~h}, \mathrm{HK}-2$ cells were stimulated with LPS for $24 \mathrm{~h}$ in the presence or absence of FF. Data are presented as the mean \pm SD of three independent experiments. ${ }^{*} \mathrm{P}<0.05$.

\section{Discussion}

FF is an old formula containing five botanical drugs for treating nephritis in the clinical setting. According to the Guidance for Industry: Botanical Drug Products published by the FDA in 2004, consistent good quality of botanical drug products is essential for the development of herbal medicines (FDA. Guidance for Industry. Botanical Drug Products. FDA, DC, Dec. 2016; www.fda.gov/downloads/drugs/guidancecomplianceregulatoryinformation/guidances/ ucm 458484.pdf). It is required that sufficient quantities of FF be prepared in a single batch from a single source of raw materials to sustain a high quality [15].

Currently, to improve the consistency of manufacturing quality control, the marker compounds of FF are measured with chromatographic technologies. As speculated, the effects of the mixture of five herbs might be the result of thousands of chemical compounds 
with synergistic or antagonistic effects. We applied bioinformatics and systems biology methods to investigate the target genes for effective treatment. Several proinflammatory cytokines and apoptosis-related genes were screened preliminarily with the bioinformatics analysis tool of BATMAN-TCM.

Here, we reported that FF attenuates the progression of murine AKI by inhibiting the inflammatory response and apoptosis of tubular epithelial cells in an LPS-induced renal injury model. The principle findings were that FF treatment partly attenuated the mortality rate of the animals, protected renal function, reduced renal pathological damage, and decreased the expression of inflammatory cytokines. FF treatment also alleviated renal tubular cellular apoptosis mediated by the mitochondrial pathway, which was partly related to cell junction function.

LPS is known to be detrimental to kidney proximal tubules and microvilli of the brush border, and is associated with the epithelial cells of peritubular capillaries [16]. On the basis on its anti-inflammatory properties, we examined the effects of FF on renal damage using a mouse model of LPS-induced AKI.

FF is expected to protect mouse kidney tissues from LPS-induced AKI efficiently according to the following TCM principles: Fangji induces diuresis to alleviate edema from the exterior with its bitter-cold flavor; Huangqi and Fuling invigorate the spleen for dieresis from the interior with its gan-dan flavor; and Guizhi and Gancao orient yang qi with their xin-gan flavor [8].

To demonstrate the molecular mechanism underlying the anti-inflammatory action of FF, the BATMAN-TCM database was used to predict target genes. These important target genes enabled us to identify the underlying mechanism. Interestingly, we observed there were some genes with a high node degree and gene-gene pairs including NOS1, NFKB1, and IL1B. GO and pathway analysis were applied to evaluate the biological functions of these target genes. It was shown that inflammatory mediator channels, cell junctions, and apoptotic process were closely related to the anti-inflammatory mechanism of FF. LPS is known to induce the secretion of inflammatory cytokines including IL-1 $\beta$ and COX-2 [17], which are closely associated with AKI [18]. Except for iNOS, NF- $\kappa B$ and IL-1 $\beta$ were detected in bioinformatics analysis, and other inflammatory-related cytokines including MCP-1, TNF- $\alpha$, and COX- 2 were also affected in an in vivo test. It was suggested that FF could improve the inflammatory milieu of the kidney.

Recent studies of AKI have focused on identifying biomarkers that are characterized as early, noninvasive, and sensitive indicators of AKI. Two of the most promising biomarkers of AKI are KIM-1 [19] and NGAL [20]. We found that pretreatment with FF inhibited the overexpression of KIM-1 and NGAL in renal tissue of LPS-induced AKI mice. The release of KIM-1 and NGAL from damaged renal tissue also leads to the downstream activation of transcription factors that regulate the expression of inflammatory cytokines and chemokines $[19,21]$.

There are intrinsic and extrinsic pathways involved in LPS-induced apoptotic processes [22]. In the classic intrinsic pathway, sepsis results in the activation of caspases [17, 23]. In the extrinsic pathway, LPS influences the Bax/Bcl-2 ratio by stimulating the secretion of a huge amount of inflammatory cytokines [24], which was supported by database analysis and further experiments in the present study.

Inflammation is a complex process that involves the dysfunction of cell-cell and cellmatrix interactions [7]. It has been reported that these pathological conditions are related to the disruption of gap junction-mediated intercellular communications [25]. Gap junctions are composed of intercellular channels formed by connexins [26]. Increasing evidence indicates the importance of connexins in modulating the severity of inflammatory disease [27]. $\mathrm{Cx} 43$ is a major vascular connexin. The upregulation of $\mathrm{Cx} 43$ has also been reported in renal inflammation in humans and rodents [28]. In the present study, Cx43 expression was almost negligible in the renal cortex of control mice. In contrast, the markedly increased expression of $\mathrm{Cx} 43$ in the kidney of the LPS-treated group was reduced by FF. This indicated that the knockdown of $\mathrm{Cx} 43$ facilitated the protective effects of $\mathrm{FF}$ against the inflammation 


\section{Cellular Physiology Cell Physiol Biochem 2018;49:2124-2137

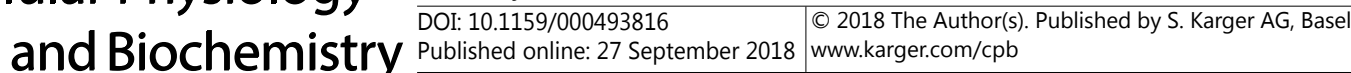 \\ Su et al.: Amelioration of LPS-Induced Renal Injury by Fangjifuling}

and apoptosis induced by LPS in HK-2 cells, suggesting that $\mathrm{Cx} 43$ is involved in the antiinflammatory and anti-apoptotic effects of FF.

\section{Conclusion}

Our study demonstrated that $\mathrm{FF}$ improves renal function and ameliorates renal histopathological changes in animals with LPS-induced AKI. The therapeutic function of FF may mainly be attributed to its effects on the production of inflammation cytokines and renal cell apoptosis and partly related to cell junction function. The clarification of the underlying mechanism of FF against LPS-induced renal injury will provide new natural renoprotective products against inflammation in AKI nephropathy.

\section{Acknowledgements}

This work was supported by National Natural Science Funds of China (No. 81703831), the Budget Project of Shanghai Municipal Education Commission (No. 2014YSN05) and Program of Supporting Young Teachers in Shanghai College, China (No. ZZSZY15003).

\section{Disclosure Statement}

All authors declare no conflict of interests.

\section{References}

1 Warnock DG: Towards a definition and classification of acute kidney injury. J Am Soc Nephrol 2005;16:3149-3150.

-2 Wang Y, Tay YC, Harris DC: Proximal tubule cells stimulated by lipopolysaccharide inhibit macrophage activation. Kidney Int 2004;66:655-662.

3 Shao Y, Fan Y, Xie Y, Yin L, Zhang Y, Deng L, Sun X, Shao X, Tan X, He J, Zhao S: Effect of continuous renal replacement therapy on kidney injury molecule-1 and neutrophil gelatinase-associated lipocalin in patients with septic acute kidney injury. Exp Ther Med 2017;13:3594-3602.

4 Nagase M, Kurihara H, Aiba A, Young MJ, Sakai T: Deletion of rac1gtpase in the myeloid lineage protects against inflammation-mediated kidney injury in mice. PloS One 2016;11:e0150886.

5 Li T, Liu Y, Zhao J, Miao S, Xu Y, Liu K, Liu M, Wang G, Xiao X: Aggravation of acute kidney injury by mpges-2 down regulation is associated with autophagy inhibition and enhanced apoptosis. Sci Rep 2017;7:10247.

6 Zhang JJ, Yu RH: [treatment of focal segmental glomerular sclerosis by modified fangji huangqi decoction: A case report of one patient]. Zhongguo Zhong Xi Yi Jie He Za Zhi 2015;35:252-253.

-7 Lin YC, Chang CW, Wu CR: Anti-nociceptive, anti-inflammatory and toxicological evaluation of fang-jihuang-qi-tang in rodents. BMC Complement Altern Med 2015;15:10.

$>8$ Wang R, Ma TM, Liu F, Gao HQ: [research progress on pharmacological action and clinical application of stephania tetrandrae radix]. Zhongguo Zhong Yao Za Zhi 2017;42:634-639.

-9 Su Z, Ye J, Qin Z, Ding X: Protective effects of madecassoside against doxorubicin induced nephrotoxicity in vivo and in vitro. Sci Rep 2015;5:18314.

10 Qin ZX, Zhu HY, Hu YH: Effects of lysophosphatidylcholine on beta-amyloid-induced neuronal apoptosis. Acta Pharmacol Sin 2009;30:388-395.

11 Shannon P, Markiel A, Ozier O, Baliga NS, Wang JT, Ramage D, Amin N, Schwikowski B, Ideker T: Cytoscape: A software environment for integrated models of biomolecular interaction networks. Genome Res 2003;13:2498-2504. 


\section{Cellular Physiology Cell Physiol Biochem 2018;49:2124-2137

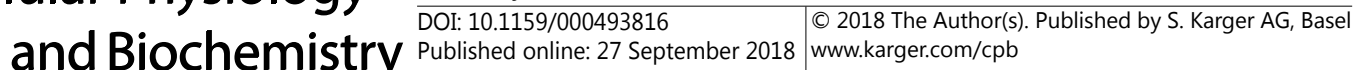

12 Han JD, Bertin N, Hao T, Goldberg DS, Berriz GF, Zhang LV, Dupuy D, Walhout AJ, Cusick ME, Roth FP, Vidal M: Evidence for dynamically organized modularity in the yeast protein-protein interaction network. Nature 2004;430:88-93.

13 Yang M, Wang B, Li M, Jiang B: Connexin 43 is involved in aldosterone-induced podocyte injury. Cell Physiol Biochem 2014;34:1652-1662.

14 Ponce A, Larre I, Castillo A, Flores-Maldonado C, Verdejo-Torres O, Contreras RG, Cereijido M: Ouabain modulates the distribution of connexin 43 in epithelial cells. Cell Physiol Biochem 2016;39:1329-1338.

15 Chen ST, Dou J, Temple R, Agarwal R, Wu KM, Walker S: New therapies from old medicines. Nat Biotechnol 2008;26:1077-1083.

16 Ikeda S, Yamamoto H, Masuda M, Takei Y, Nakahashi O, Kozai M, Tanaka S, Nakao M, Taketani Y, Segawa $\mathrm{H}$, Iwano M, Miyamoto K, Takeda E: Downregulation of renal type iia sodium-dependent phosphate cotransporter during lipopolysaccharide-induced acute inflammation. Am J Physiol Renal Physiol 2014;306:F744-750.

17 Wicki S, Gurzeler U, Wei-Lynn Wong W, Jost PJ, Bachmann D, Kaufmann T: Loss of xiap facilitates switch to tnfalpha-induced necroptosis in mouse neutrophils. Cell Death Dis 2016;7:e2422.

18 Chen L, Liu P, Feng X, Ma C: Salidroside suppressing lps-induced myocardial injury by inhibiting rosmediated pi3k/akt/mtor pathway in vitro and in vivo. J Cell Mol Med 2017;21:3178-3189.

19 Kokura K, Kuromi Y, Endo T, Anzai N, Kazuki Y, Oshimura M, Ohbayashi T: A kidney injury molecule-1 (kim-1) gene reporter in a mouse artificial chromosome: The responsiveness to cisplatin toxicity in immortalized mouse kidney s3 cells. J Gene Med 2016;18:273-281.

20 Kai K, Yamaguchi T, Yoshimatsu Y, Kinoshita J, Teranishi M, Takasaki W: Neutrophil gelatinase-associated lipocalin, a sensitive urinary biomarker of acute kidney injury in dogs receiving gentamicin. J Toxicol Sci 2013;38:269-277.

21 Zager RA, Johnson AC, Lund S: Uremia impacts renal inflammatory cytokine gene expression in the setting of experimental acute kidney injury. Am J Physiol Renal Physiol 2009;297:F961-970.

-22 Martin EL, Ranieri VM: Endotoxin removal by polymyxin b immobilized cartridge inactivates circulating proapoptotic factors. Contrib Nephrol 2010;167:65-76.

-23 Duranton C, Rubera I, L’Hoste S, Cougnon M, Poujeol P, Barhanin J, Tauc M: Kcnq1 k+ channels are involved in lipopolysaccharide-induced apoptosis of distal kidney cells. Cell Physiol Biochem 2010;25:367-378.

-24 Cantaluppi V, Assenzio B, Pasero D, Romanazzi GM, Pacitti A, Lanfranco G, Puntorieri V, Martin EL, Mascia L, Monti G, Casella G, Segoloni GP, Camussi G, Ranieri VM: Polymyxin-b hemoperfusion inactivates circulating proapoptotic factors. Intensive Care Med 2008;34:1638-1645.

25 Chanson M, Derouette JP, Roth I, Foglia B, Scerri I, Dudez T, Kwak BR: Gap junctional communication in tissue inflammation and repair. Biochim Biophys Acta 2005;1711:197-207.

-26 Beyer EC, Berthoud VM: Gap junction gene and protein families: Connexins, innexins, and pannexins. Biochim Biophys Acta 2017;1860:5-8.

27 Scheckenbach KE, Crespin S, Kwak BR, Chanson M: Connexin channel-dependent signaling pathways in inflammation. J Vasc Res 2011;48:91-103.

-28 Kavvadas P, Abed A, Poulain C, Authier F, Labejof LP, Calmont A, Afieri C, Prakoura N, Dussaule JC, Chatziantoniou C, Chadjichristos CE: Decreased expression of connexin 43 blunts the progression of experimental gn. J Am Soc Nephrol 2017;28:2915-2930. 